\title{
TIME INTEGRATION OF STOCHASTIC GENERALIZED EQUATIONS OF MOTION USING SSFEM
}

\author{
MARIUSz POŃSKI \\ Czestochowa University of Technology, Department of Civil Engineering, Częstochowa, Poland \\ e-mail: mponski@bud.pcz.czest.pl
}

\begin{abstract}
The paper develops an integration approach to stochastic nonlinear partial differential equations (SPDE's) with parameters to be random fields. The methodology is based upon assumption that random fields are from a special class of functions, and can be described as a product of two functions with dependent and independent random variables. Such an approach allows one to use Karhunen-Loève expansion directly, and the modified stochastic spectral finite element method (SSFEM). It is assumed that a random field is stationary and Gaussian while the autocovariance function is known. A numerical example of onedimensional heat waves analysis is shown.
\end{abstract}

Keywords: spectral stochastic finite element method, time integration, heat waves

\section{Introduction}

In the literature, one can find works describing SPDE's solutions using SSFEM for stationary problems (Le Maitre and Knio, 2010; Matthies and Keese, 2005). There are also methods handling transient problems such as the Monte Carlo method (MC), perturbation method (Kamiński, 2013; Służalec, 2003), stochastic collocation method (SCM) (Acharjee and Zabaras, 2006; Babuška et al., 2007; Xiu and Hesthaven, 2005). These methods are widely used for many problems, e.g. continuum mechanics, fluid dynamics, heat flow and have their advantages and disadvantages (Stefanou, 2009; Xiu, 2010). As the leading in the literature, the SCM method is listed due to the possibility of analyzing complex nonlinear problems. It reduces analysis time by using multithreading and can be simply implemented (deterministic solver is treated as so-called "black box"). Competitive to SCM is the method of Stochastic Spectral Finite Element Method proposed by Ghanem and Spanos (2003). This method is one of the so-called intrusive methods, which is very effective in solving linear problems, but requires building the source code from scratch. An important disadvantage that is mentioned in many works is the coupling of equations that prevent the use of parallel solvers. This problem was solved by applying the domain decomposition method (Subber and Sarkar, 2014). Another important disadvantage mentioned in the works on numerical solution with SSFEM is the considerable difficulty of solving nonlinear problems. Mathematical formulation of nonlinear stationary equations can be found in works (Arregui-Mena et al., 2016; Ghosh et al., 2008; Hu et al., 2015; Matthies and Keese, 2005; Nouy, 2008; Nouy and Le Maitre, 2009; Stefanou et al., 2017; Xiu and Karniadakis, 2003; Zakian and Khaji, 2016) rather than description of a general numerical approach. There is also no comprehensive solution to transient problems. This paper presents a methodology based upon a special class of functions occurring in constitutive equations which can be described as a product of two functions, respectively with dependent and independent variables. This approach allows one to extend the applicability of SSFEM to solve wide range of nonstationary nonlinear stochastic PDE's. 


\section{Stochastic description}

In this work, a modified SSFEM is used. This method is based upon notion of a random field. The random field $\alpha(x, \omega)$ (Acharjee and Zabaras, 2007; Xiu, 2010) $(x \in \mathbb{D} \subset \mathbb{R}, \omega \in \Omega$ ) is a real valued measurable function which assigns a random variable $\alpha(\omega)$ to each point $x$ on a fixed probability space $(\Omega, \mathbb{Z}, \mathbb{P})$. Here $\Omega$ is the set of elementary events, $\mathbb{Z}$ is the $\sigma$-algebra and $\mathbb{P}: \mathbb{Z} \rightarrow[0,1]$ is a probability measure. To obtain a computationally useful representation of the process $\alpha(x, \omega)$, it will be presented in the canonical form. Among various forms of such a representation, a spectral representation - Karhunen-Loève expansion will be adopted in further considerations (Ghanem and Spanos, 2003). This expansion may be presented in the following form

$$
\alpha(x, \omega)=\xi_{0} \overline{\alpha(x)}+\sum_{i=1}^{\infty} \xi_{i}(\omega) \sqrt{\lambda_{i}} f_{i}(x) \quad x \in \mathbb{D}, \omega \in \Omega
$$

Such a Karhunen-Loève expansion is truncated to $M$ terms

$$
\alpha(x, \omega) \approx \xi_{0} \overline{\alpha(x)}+\sum_{i=1}^{M} \xi_{i}(\omega) \sqrt{\lambda_{i}} f_{i}(x) \quad x \in \mathbb{D}, \omega \in \Omega
$$

In equation (2.1), $\left\{\xi_{i}(\omega)\right\}_{i=1}^{\infty}$ is a set of othonormal independent Gaussian random variables with mean $\xi_{0}=1$ and standard deviations equal to one, $\overline{(\cdot)}$ is the expected value operator. Constants $\left\{\lambda_{i}\right\}_{i=1}^{\infty}$ and deterministic functions $\left\{f_{i}(x)\right\}_{i=1}^{\infty}$ are the eigenvalues and eigenfunctions of the covariance kernel

$$
\int_{\mathbb{D}} C_{\text {kernel }}\left(x_{1}, x_{2}\right) f_{i}\left(x_{2}\right) d x_{2}=\lambda_{i} f_{i}\left(x_{1}\right) \quad i \in \mathbb{N}=\{1,2, \ldots\}
$$

The polynomial chaos (Ghanem and Spanos, 2003; Le Maitre and Knio, 2010) representation of the random variable $U(\omega)$, truncated to $P$ terms, can be written as

$$
U(\omega) \approx U(\boldsymbol{\xi})=\sum_{i=0}^{P} u_{i} \Phi_{i}(\boldsymbol{\xi})
$$

where $\left\{\Phi_{i}(\boldsymbol{\xi})\right\}_{i=0}^{P}$ denotes polynomial chaoses (Ghanem and Spanos, 2003), and $\left\{u_{i}\right\}_{i=0}^{P}$ are coefficients of the expansion. The coefficient $P$ described by the expression (Ghanem and Spanos, 2003)

$$
P=1+\sum_{s=1}^{p} \frac{2}{s !} \prod_{r=0}^{s-1}(M+r)
$$

is the total number of polynomial chaoses used in the expansion, excluding the zero-th order term, with $p$ denoting the order of polynomial chaoses (detailed description of polynomial chaoses can be found in Ghanem and Spanos (2003), Le Maitre and Knio (2010)).

\section{Governing equations formulation of SSFEM - an approach for a special class of equations}

\subsection{Method of solution of the stochastic problem (SSFEM)}

First step in solving SPDE's, after finite element discretisation, is the stochastic random field discretisation. Let $K$ denotes the number of nodes of the discretized domain. The equation of 
motion with given initial and boundary conditions, which is analyzed, can be written in a well known matrix form (Bathe, 1996)

$$
\mathbf{M}(\mathbf{x}, \omega, \mathbf{q}(t, \omega)) \ddot{\mathbf{q}}(t, \omega)+\mathbf{C}(\mathbf{x}, \omega, \mathbf{q}(t, \omega)) \dot{\mathbf{q}}(t, \omega)+\mathbf{K}(\mathbf{x}, \omega, \mathbf{q}(t, \omega)) \mathbf{q}(t, \omega)=\mathbf{F}(\mathbf{x}, \omega, \mathbf{q}(t, \omega))
$$

where $t \in \mathbb{T}$ denotes time, $\mathbf{x} \in \mathbb{D} \times \mathbb{D} \times \mathbb{D} \stackrel{\text { def }}{=} \mathbb{D}^{3} \subset \mathbb{R}^{3}$ denotes space variables, $\mathbf{q}(t, \omega)$ is a generalized $K \times 1$ displacement vector and $\dot{\mathbf{q}}(t, \omega), \ddot{\mathbf{q}}(t, \omega)$ is the first and second derivative in time. Suppose that for the $K \times K$ matrices $\mathbf{M}, \mathbf{C}, \mathbf{K}$ and $K \times 1$ vector $\mathbf{F}$ separation of dependent and independent variables can be made, which allows one to use Karhunen-Loève expansion directly, e.g.

$$
\mathbf{K}(\mathbf{x}, \omega, \mathbf{q}(t, \omega))=\mathbf{K}(\mathbf{x}, \omega) f_{K}(\mathbf{q}(t, \omega))
$$

where $f_{K}(\mathbf{q}(t, \omega))$ is a real valued Riemann integrable function on a suitable space and $\mathbf{K}(\mathbf{x}, \omega)$ is a $K \times K$ matrix. Such an approach limits applicability of this method to problems in which constitutive equations or nonlinear boundary conditions can be written as a product of functions of independent and dependent variables, e.g. $k(\mathbf{x}, q(\mathbf{x}, t))=k_{a}(\mathbf{x}) k_{b}(q(\mathbf{x}, t))$. Many physical relations can be written in this way or can be reduced to either $k(\mathbf{x}, q(\mathbf{x}, t))=k_{b}(q(\mathbf{x}, t))$ or $k(\mathbf{x}, q(\mathbf{x}, t))=k_{a}(\mathbf{x})$.

Assume that the discretized function of generalized displacement at each node has representations in polynomial chaos

$$
q_{k}(t, \omega) \approx \sum_{i=0}^{P}\left(q_{\text {spect }}(t)\right)_{k, i} \Phi_{i}(\boldsymbol{\xi}(\omega)) \quad k=1,2, \ldots, K
$$

(index $k$ denotes node number) and let it be derived its first and second order derivative with respect to the time

$$
\begin{array}{ll}
\dot{q}_{k}(t, \omega) \approx \sum_{i=0}^{P}\left(\dot{q}_{\text {spect }}(t)\right)_{k, i} \Phi_{i}(\boldsymbol{\xi}(\omega)) & k=1,2, \ldots, K \\
\ddot{q}_{k}(t, \omega) \approx \sum_{i=0}^{P}\left(\ddot{q}_{\text {spect }}(t)\right)_{k, i} \Phi_{i}(\boldsymbol{\xi}(\omega)) & k=1,2, \ldots, K
\end{array}
$$

The vector of nodal generalized displacement can be written as

$$
\mathbf{q}(t, \omega) \approx \mathbf{q}(t, \boldsymbol{\xi})=\mathbf{q}_{\text {spect }}^{\text {matrix }}(t) \boldsymbol{\Phi}_{\text {node }}(\boldsymbol{\xi})
$$

where $\mathbf{q}_{\text {spect }}^{\text {matrix }}(t)$ is a $K \times(P+1)$ matrix built from spectral coefficients and

$$
\boldsymbol{\Phi}_{\text {node }}(\boldsymbol{\xi})=\left[\begin{array}{llll}
\Phi_{0}(\boldsymbol{\xi}) & \Phi_{1}(\boldsymbol{\xi}) & \cdots & \Phi_{P}(\boldsymbol{\xi})
\end{array}\right]^{\mathrm{T}}
$$

is a vector built from the polynomial chaoses.

Substituting appropriate derivatives of equation (3.5) to equation (3.1) and using (3.2), (3.5)-(3.6), the following equation

$$
\begin{aligned}
& \mathbf{M}(\mathbf{x}, \omega) f_{M}\left(\mathbf{q}_{\text {spect }}^{\text {matrix }}(t) \boldsymbol{\Phi}_{\text {node }}(\boldsymbol{\xi})\right) \ddot{\mathbf{q}}_{\text {spect }}^{\text {matrix }}(t) \boldsymbol{\Phi}_{\text {node }}(\boldsymbol{\xi}) \\
& \quad+\mathbf{C}(\mathbf{x}, \omega) f_{C}\left(\mathbf{q}_{\text {spect }}^{\text {matrix }}(t) \boldsymbol{\Phi}_{\text {node }}(\boldsymbol{\xi})\right) \dot{\mathbf{q}}_{\text {spect }}^{\text {matrix }}(t) \boldsymbol{\Phi}_{\text {node }}(\boldsymbol{\xi}) \\
& \quad+\mathbf{K}(\mathbf{x}, \omega) f_{K}\left(\mathbf{q}_{\text {spect }}^{\text {matrix }}(t) \boldsymbol{\Phi}_{\text {node }}(\boldsymbol{\xi})\right) \mathbf{q}_{\text {spect }}^{\text {matrix }}(t) \boldsymbol{\Phi}_{\text {node }}(\boldsymbol{\xi})=\mathbf{F}(\mathbf{x}, \omega) f_{F}\left(\mathbf{q}_{\text {spect }}^{\text {matrix }}(t)\right)
\end{aligned}
$$

can be obtained. 
Let us represent the matrices $\mathbf{M}(\mathbf{x}, \omega), \mathbf{C}(\mathbf{x}, \omega), \mathbf{K}(\mathbf{x}, \omega), \mathbf{F}(\mathbf{x}, \omega)$ using the Karhunen-Loève expansion, e.g.

$$
\mathbf{K}(\mathbf{x}, \omega) \approx \mathbf{K}(\mathbf{x}, \boldsymbol{\xi})=(\mathbf{K}(\mathbf{x}))_{0} \xi_{0}+\sum_{i=1}^{M} \xi_{i}(\omega)\left(\mathbf{K}^{0}(\mathbf{x})\right)_{i}
$$

where $(\cdot)_{0}$ denotes a matrix computed for the mean value of the process and $(\cdot)^{0}$ denotes a matrix built of the shape functions and Karhunen-Loève expansion terms (e.g. $\left(\mathbf{K}^{0}(\mathbf{x})\right)_{i}=$ $\int_{\mathbb{D}} \nabla \mathbf{N}\left(\sqrt{\lambda_{i}} f_{i}(x)\right) \nabla \mathbf{N}^{\mathrm{T}} d \mathbf{x}$, where $\mathbf{N}$ is a vector built of test functions from the Sobolev space $H^{1}\left(0, l_{\text {elem }}\right)$ where $l_{\text {elem }}$ is a finite element length $)$.

In order to formulate a suitable system of equations, let us represent the matrix of stochastic eigenmodes of the solution as a vector $\mathbf{q}_{\text {spect }}^{\text {matrix }} \rightarrow \mathbf{q}_{\text {spect }}^{\text {vector }}$ where

$$
\mathbf{q}_{\text {spect }}^{\text {vector }}(t)=\left[\begin{array}{llll}
{\left[q_{00}(t)\right.} & q_{01}(t) & \cdots & q_{0 P}(t)
\end{array}\right]\left[\begin{array}{lllll}
\cdots & q_{K 0}(t) & \cdots & q_{K P}(t)
\end{array}\right]^{\mathrm{T}}
$$

where $P$, as before, is the total number of polynomial chaoses used in the expansion and $K$ is the total number of nodes in the FEM solution. The global vector of polynomial chaoses takes the form

$$
\boldsymbol{\Phi}(\boldsymbol{\xi})=\left[\begin{array}{llll}
\left(\boldsymbol{\Phi}_{\text {node }}(\boldsymbol{\xi})\right)_{0} & \left(\boldsymbol{\Phi}_{\text {node }}(\boldsymbol{\xi})\right)_{1} & \cdots & \left(\boldsymbol{\Phi}_{\text {node }}(\boldsymbol{\xi})\right)_{K}
\end{array}\right]^{\mathrm{T}}
$$

where the vectors of polynomial chaoses are the same for each node

$$
\left(\boldsymbol{\Phi}_{\text {node }}(\boldsymbol{\xi})\right)_{0}=\left(\boldsymbol{\Phi}_{\text {node }}(\boldsymbol{\xi})\right)_{1}=\ldots=\left(\boldsymbol{\Phi}_{\text {node }}(\boldsymbol{\xi})\right)_{K}
$$

After substitution of appropriate expansions (3.8) of the matrices $\mathbf{M}(\mathbf{x}, \omega), \mathbf{C}(\mathbf{x}, \omega), \mathbf{K}(\mathbf{x}, \omega)$, $\mathbf{F}(\mathbf{x}, \omega)$, equation (3.9) and (3.10) into (3.7), then multiplying by $\boldsymbol{\Phi}(\boldsymbol{\xi})$ and averaging with respect to the random space

$$
\begin{aligned}
& \left\langle\boldsymbol{\Phi}(\boldsymbol{\xi})\left((\mathbf{M}(\mathbf{x}))_{0} \xi_{0}+\sum_{i_{M}=1}^{M_{M}} \xi_{i_{M}}\left(\mathbf{M}^{0}(\mathbf{x})\right)_{i_{M}}\right) f_{M_{T}}\left(\left(\mathbf{q}_{\text {spect }}^{\text {vector }}(t)\right)^{\mathrm{T}} \mathbf{\Phi}(\boldsymbol{\xi})\right)\left(\ddot{\mathbf{q}}_{\text {spect }}^{\text {vector }}(t)\right)^{\mathrm{T}} \mathbf{\Phi}(\boldsymbol{\xi})\right\rangle \\
& +\left\langle\boldsymbol{\Phi}(\boldsymbol{\xi})\left((\mathbf{C}(\mathbf{x}))_{0} \xi_{0}+\sum_{i_{C}=1}^{M_{C}} \xi_{i_{C}}\left(\mathbf{C}^{0}(\mathbf{x})\right)_{i_{C}}\right) f_{C_{T}}\left(\left(\mathbf{q}_{\text {spect }}^{\text {vector }}(t)\right)^{\mathrm{T}} \mathbf{\Phi}(\boldsymbol{\xi})\right)\left(\dot{\mathbf{q}}_{\text {spect }}^{\text {vector }}(t)\right)^{\mathrm{T}} \mathbf{\Phi}(\boldsymbol{\xi})\right\rangle \\
& +\left\langle\boldsymbol{\Phi}(\boldsymbol{\xi})\left((\mathbf{K}(\mathbf{x}))_{0} \xi_{0}+\sum_{i_{K}=1}^{M_{K}} \xi_{i_{K}}\left(\mathbf{K}^{0}(\mathbf{x})\right)_{i_{K}}\right) f_{K_{T}}\left(\left(\mathbf{q}_{\text {spect }}^{\text {vector }}(t)\right)^{\mathrm{T}} \mathbf{\Phi}(\boldsymbol{\xi})\right)\left(\mathbf{q}_{\text {spect }}^{\text {vector }}(t)\right)^{\mathrm{T}} \mathbf{\Phi}(\boldsymbol{\xi})\right\rangle \\
& =\left\langle\boldsymbol{\Phi}(\boldsymbol{\xi})\left((\mathbf{F}(\mathbf{x}))_{0} \xi_{0}+\sum_{i_{F}=1}^{M_{F}} \xi_{i_{F}}\left(\mathbf{F}^{0}(\mathbf{x})\right)_{i_{F}}\right) f_{F}\left(\left(\mathbf{q}_{\text {spect }}^{\text {vector }}(t)\right)^{\mathrm{T}} \mathbf{\Phi}(\boldsymbol{\xi})\right)\right\rangle
\end{aligned}
$$

can be obtained, where

$$
\langle\cdot\rangle=\int_{\Omega}(\cdot) d \mathbb{P}(\omega)
$$

and $M_{M}, M_{C}, M_{K}, M_{F}$ denote the numbers of terms in the Karhunen-Loève expansion.

Finally, the set of $(P+1) K$ nonlinear deterministic equations of the SSFEM method is obtained

$$
\begin{gathered}
\mathbf{M}^{\text {expand }}\left(\mathbf{x}, \mathbf{q}_{\text {spect }}^{\text {vect }}(t)\right) \ddot{\mathbf{q}}_{\text {spect }}^{\text {vect }}(t)+\mathbf{C}^{\text {expand }}\left(\mathbf{x}, \mathbf{q}_{\text {spect }}^{\text {vect }}(t)\right) \dot{\mathbf{q}}_{\text {spect }}^{\text {vect }}(t) \\
+\mathbf{K}^{\text {expand }}\left(\mathbf{x}, \mathbf{q}_{\text {spect }}^{\text {vector }}(t)\right) \mathbf{q}_{\text {spect }}^{\text {vector }}(t)=\mathbf{F}^{\text {expand }}\left(\mathbf{x}, \mathbf{q}_{\text {spect }}^{\text {vector }}(t)\right)
\end{gathered}
$$


where, for example, the generalized stiffness matrix (matrices $\mathbf{M}^{\text {expand }}$ and $\mathbf{C}^{\text {expand }}$ can be written in the same way)

$$
\begin{aligned}
& \mathbf{K}^{\text {expand }}\left(\mathbf{x}, \mathbf{q}_{\text {spect }}^{\text {vector }}(t)\right) \\
& \quad=\left\langle\boldsymbol{\Phi}(\boldsymbol{\xi})\left((\mathbf{K}(\mathbf{x}))_{0} \xi_{0}+\sum_{i_{K}=1}^{M_{K}} \xi_{i}\left(\mathbf{K}^{0}(\mathbf{x})\right)_{i_{K}}\right) f_{K}\left(\left(\mathbf{q}_{\text {spect }}^{\text {vector }}(t)\right)^{\mathrm{T}} \boldsymbol{\Phi}(\boldsymbol{\xi})\right)(\boldsymbol{\Phi}(\boldsymbol{\xi}))^{\mathrm{T}}\right\rangle
\end{aligned}
$$

and the generalized force vector

$$
\mathbf{F}^{\text {expand }}\left(\mathbf{x}, \mathbf{q}_{\text {spect }}^{\text {vector }}(t)\right)=\left\langle\boldsymbol{\Phi}(\boldsymbol{\xi})\left((\mathbf{F}(\mathbf{x}))_{0} \xi_{0}+\sum_{i_{F}=1}^{M_{F}} \xi_{i}\left(\mathbf{F}^{0}(\mathbf{x})\right)_{i_{F}}\right) f_{F}\left(\left(\mathbf{q}_{\text {spect }}^{\text {vector }}(t)\right)^{\mathrm{T}} \mathbf{\Phi}(\boldsymbol{\xi})\right)\right\rangle
$$

For $f_{r}\left(\mathbf{q}^{\mathrm{T}} \mathbf{\Phi}(\boldsymbol{\xi})\right)=1, r=M, C, K, F$ equation (3.14) is linear. Moreover, the terms $\left\langle\xi_{i} \boldsymbol{\Phi}(\boldsymbol{\xi})(\boldsymbol{\Phi}(\boldsymbol{\xi}))^{\mathrm{T}}\right\rangle$ and $\left\langle\xi_{i} \boldsymbol{\Phi}(\boldsymbol{\xi})\right\rangle$ of the above stated matrices obtained from integration over the random space have a lot of zero entries (Ghanem and Spanos, 2003). In addition, this terms may be determined in advance and only once.

In the proposed solution of nonlinear equation (3.14), the matrices in this equation have to be numerically integrated both in the iteration step and time step, over a random and geometric space. This is due to nonlinear functions $f_{r}$ (where $r=M, C, K, F$ ) of the dependent variable $q$ appearing in the parts $\left\langle\xi_{i} \boldsymbol{\Phi}(\boldsymbol{\xi}) f_{g}\left(\mathbf{q}^{\mathrm{T}} \boldsymbol{\Phi}(\boldsymbol{\xi})\right)(\boldsymbol{\Phi}(\boldsymbol{\xi}))^{\mathrm{T}}\right\rangle$ (where $g=M, C, K$ ) and $\left\langle\xi_{i} \boldsymbol{\Phi}(\boldsymbol{\xi}) f_{F}\left(\mathbf{q}^{\mathrm{T}} \boldsymbol{\Phi}(\boldsymbol{\xi})\right)\right\rangle$ of these matrices. Methods of evaluating the above mentioned inner product for different types of nonlinearities of the functions $f_{r}$ can be found in work of Le Maitre and Knio (2010).

For a complete presentation of the stochastic process $q(\mathbf{x}, t, \omega)$, the covariance matrix is determined (Ghanem and Spanos, 2003)

$$
\operatorname{Cov}_{q q}(t)=\sum_{j=0}^{P}\left\langle\Phi_{j}(\boldsymbol{\xi}) \Phi_{j}(\boldsymbol{\xi})\right\rangle\left(\mathbf{q}_{\text {spect }}^{\text {vector }}(t)\right)_{j}\left[\left(\mathbf{q}_{\text {spect }}^{\text {vector }}(t)\right)_{j}\right]^{\mathrm{T}}
$$

where $\operatorname{Cov}_{q q}=\left\{\left(\operatorname{Cov}_{q q}\right)_{i, j}\right\}_{i, j=1}^{P}$, the $j$-th eigenmode of the vector $\mathbf{q}_{\text {spect }}^{\text {vector }}(t)$ has been written as $\left(\mathbf{q}_{\text {spect }}^{\text {vector }}(t)\right)_{j}$. Therefore, the expected value may be calculated

$$
E\left(\mathbf{q}_{\text {spect }}^{\text {vector }}(t)\right)=\left(\mathbf{q}_{\text {spect }}^{\text {vector }}(t)\right)_{0}
$$

and the variance

$$
\operatorname{Var}_{q}(t)_{i}=\operatorname{Cov}_{q q}(t)_{i, i} \quad i=1,2, \ldots, P
$$

Matrix equation (3.14) is a deterministic system of nonlinear equations, therefore, in order to solve it, one of methods of direct integration, for example Newmark method, can be used (Bathe, 1996).

\subsection{Application of SSFEM to non-classical stochastic heat conduction constitutive model - heat waves analysis}

The most widely used model for many engineering problems is the classic equation of Fourier (Fourier, 1822), which can be represented as a function of a random field. The expression for the heat flux can be written

$$
\mathbf{q}_{F}(\mathbf{x}, t, \omega)=-k_{F}(\mathbf{x}, \omega, T) \nabla T(\mathbf{x}, t, \omega) \quad \mathbf{x} \in \mathbb{D}^{3} \subset \mathbb{R}^{3}, \omega \in \Omega, \quad t \in \mathbb{T}
$$

where $k_{F}(\mathbf{x}, \omega, T)$ is Fourier thermal conductivity. 
Because of the anomalies associated with the Fourier model (Vernotte, 1958) and the presence of finite speed propagation of heat, a Cattaneo model that takes into account heat flux relaxation has been introduced (Cattaneo, 1948; Służalec, 2003)

$$
\tau \partial_{t} \mathbf{q}_{C}(\mathbf{x}, t, \omega)+\mathbf{q}_{C}(\mathbf{x}, t, \omega)=-k_{C}(\mathbf{x}, \omega, T) \nabla T(\mathbf{x}, t, \omega) \quad \mathbf{x} \in \mathbb{D}^{3} \subset \mathbb{R}^{3}, \omega \in \Omega, \quad t \in \mathbb{T}
$$

where $\tau$ represents the relaxation time and $k_{C}(\mathbf{x}, \omega, T)$ is the Cattaneo thermal conductivity.

The Jeffreys type model is another heat conduction constitutive model of which the Cattaneo model and a Fourier-like diffusive model are subcases that can be obtained from this model (Joseph and Preziosi, 1989; Straughan, 2011; Tamma and Zhou, 1998; Ván and Fülöp, 2012)

$$
\tau \partial_{t} \mathbf{q}(\mathbf{x}, t, \omega)+\mathbf{q}(\mathbf{x}, t, \omega)=-k(\mathbf{x}, \omega, T)\left[\nabla T(\mathbf{x}, t, \omega)-K(\mathbf{x}, \omega, T) \partial_{t}(\nabla T(\mathbf{x}, t, \omega))\right]
$$

where $\mathbf{x} \in \mathbb{D}^{3} \subset \mathbb{R}^{3}, \omega \in \Omega, t \in \mathbb{T}$, and

$$
k(\mathbf{x}, \omega, T)=k_{F}(\mathbf{x}, \omega, T)+k_{C}(\mathbf{x}, \omega, T)
$$

and

$$
K(\mathbf{x}, \omega, T)=\frac{\tau k_{F}(\mathbf{x}, \omega, T)}{k(\mathbf{x}, \omega, T)}
$$

is the so-called retardation time.

When the retardation time, $K=0$, the Jeffreys model is reduced to the Cattaneo model. When selecting, $K=\tau$, the Jeffreys model only degenerates to a Fourier-like diffusive model with relaxation (Tamma and Zhou, 1998).

Zhou and co-workers (Tamma and Zhou, 1998) introduced C-process and F-process models which are a linear combination of the Fourier and Cattaneo models. The basic assumption is the simultaneous occurrence of a fast process based on equation (3.21) and a slow process related to equation (3.22). This model is a generalization of the above stated relations. The equations describing connection between heat flux and temperature have the following form (Tamma and Zhou, 1998)

$$
\mathbf{q}_{C F}(\mathbf{x}, t, \omega)=\mathbf{q}_{F}(\mathbf{x}, t, \omega)+\mathbf{q}_{C}(\mathbf{x}, t, \omega) \quad \mathbf{x} \in \mathbb{D}^{3} \subset \mathbb{R}^{3}, \omega \in \Omega, \quad t \in \mathbb{T}
$$

where

$$
\begin{aligned}
& \mathbf{q}_{C}(\mathbf{x}, t, \omega)+\tau \partial_{t} \mathbf{q}_{C}(\mathbf{x}, t, \omega)=-\left(1-F_{T}(\mathbf{x}, \omega, T)\right) k(\mathbf{x}, \omega, T) \nabla T(\mathbf{x}, t, \omega) \\
& \mathbf{q}_{F}(\mathbf{x}, t, \omega)=-F_{T}(\mathbf{x}, \omega, T) k(\mathbf{x}, \omega, T) \nabla T(\mathbf{x}, \omega) \\
& F_{T}(\mathbf{x}, \omega, T)=\frac{k_{F}(\mathbf{x}, \omega, T)}{k_{F}(\mathbf{x}, \omega, T)+k_{C}(\mathbf{x}, \omega, T)} \\
& k(\mathbf{x}, \omega, T)=k_{F}(\mathbf{x}, \omega, T)+k_{C}(\mathbf{x}, \omega, T)
\end{aligned}
$$

After substitution of equation (3.25) 1,2 into (3.24), it can be obtained

$$
\begin{aligned}
& \mathbf{q}_{C F}(\mathbf{x}, t, \omega)+\tau \partial_{t} \mathbf{q}_{C F}(\mathbf{x}, t, \omega) \\
& \quad=-\left[k(\mathbf{x}, \omega, T) \nabla T(\mathbf{x}, t, \omega)+\tau \partial_{t}\left(k(\mathbf{x}, \omega, T) F_{T}(\mathbf{x}, \omega, T) \nabla T(\mathbf{x}, t, \omega)\right)\right]
\end{aligned}
$$

Indexes $F$ and $C$ in the conductivity coefficient and in the heat flux vector respectively refer to the Fourier model with infinite propagation speed of wave and to the Cattaneo model, occurring simultaneously. Also the model number $F_{T} \in[0,1]$ has been introduced. It can be seen that for $F_{T} \in[0,1]$ the Jeffrey model is obtained, for $F_{T}=1$ the Fourier one, and for $F_{T}=0$ the equation is reduced to the Cattaneo model. 


\section{Stochastic nonlinear 1D transport equation}

To obtain equations describing the flow of heat in a rigid conductor, the following energy balance equation (for clarity the shorthand notation is adopted $T \equiv T(x, t, \omega)$ )

$$
C(x, \omega, T) \partial_{t} T+\nabla \cdot \mathbf{q}_{C F}=0 \quad x \in \mathbb{D}, \omega \in \Omega, \quad t \in \mathbb{T}
$$

where $C(x, \omega, T)=\rho c(x, \omega, T)$ is combined with one of the model constitutive equation, initial condition $T(x, t=0, \omega)=T_{0}$ and a suitable boundary condition on $\partial \mathbb{D}$. The equation associated with $\mathrm{C}$ - and F-process models (which can be reduced to the above mentioned models) is stated below

$$
\begin{aligned}
& \tau \partial_{t}\left(C(x, \omega, T) \partial_{t} T\right)+C(x, \omega, T) \partial_{t} T-\partial_{x}\left(k_{F}(x, \omega, T) \partial_{x} T\right)-\tau \partial_{t}\left(\partial_{x}\left(k_{F}(x, \omega, T) \partial_{x} T\right)\right) \\
& \quad-\partial_{x}\left(\left(1-F_{T}(x, \omega, T)\right) k(x, \omega, T) \partial_{x} T\right)=0
\end{aligned}
$$

\section{Governing equations formulation of the Galerkin Finite Element Method (GFEM) - C- and F-processes model}

The first step to solve the stochastic problem is discretisation of a deterministic space by the use of the finite element method in the Galerkin approach (Bathe, 1996). To this end, the response in terms of the temperature field is approximated by the expression

$$
T(\mathbf{x}, t, \omega)=(\mathbf{T}(t, \omega))^{\mathrm{T}} \mathbf{N}(\mathbf{x})
$$

where $\mathbf{N}(\mathbf{x})$ is a vector built of the test functions (as defined in Section 3.1), $\mathbf{T}(t, \omega)$ is a vector representing the discretized temperature field and the upper index $\mathrm{T}$ denotes transposition. The final equations after appropriate transformations read

$$
\mathbf{M}(\mathbf{T}) \ddot{\mathbf{T}}+\mathbf{C}(\mathbf{T}) \dot{\mathbf{T}}+\mathbf{K}(\mathbf{T}) \mathbf{T}=\mathbf{F}(\mathbf{T})
$$

where $\dot{\mathbf{T}}$ and $\ddot{\mathbf{T}}$ denote the first and second order temperature derivative with respect to time. The individual matrices in equation (5.2) can be obtained through a standard FE method (Bathe, 1996).

\section{Numerical example}

Distributions of temperature statistical moments as functions of time of the considered model for a thin steel sheet (similar to the work (Al-Nimr, 1997)) (Fig. 1) heated by a sudden heat impulse (Ván and Fülöp, 2012) will be analyzed. For this purpose and for further analysis, the following data will be adopted (Joseph and Preziosi, 1989; Ván and Fülöp, 2012).

Pulsed heating can be modeled as an internal heat source (Bargmann and Favata, 2014) with various time characteristics or as external boundary conditions. Let the heating function for the mixed boundary condition (convection-radiation) takes the form

$$
T_{\text {pulse }}(t)=T_{0}+a \sin (b t) \exp (-c t)
$$

with parameters $T_{0}=293.15 \mathrm{~K}, a=15 \cdot 10^{4} \mathrm{~K}, b=\pi / 10, c=50$.

One dimensional region of the sample (modeled as a bar) is divided into 20 elements. The adopted heating time is equal to $t_{\max }=0.6 \cdot 10^{-10} \mathrm{~s}$ with 60 time steps (time increment $\Delta t=0.01$. $\left.10^{-10}\right)$. Cattaneo thermal conductivity has been considered as a random function independent 


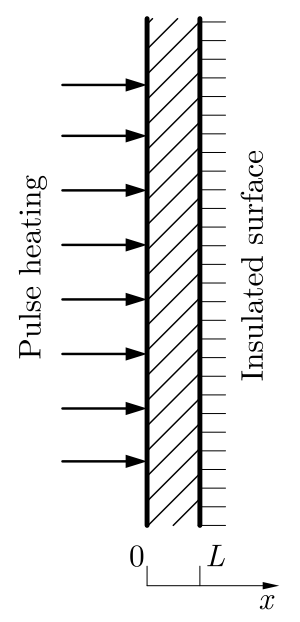

Fig. 1. Schematic of a thin steel plate heated by a pulse

of temperature (stationary and Gaussian process), $k_{C}(x, \omega, T)=k_{C}(x, \omega)$ with the covariance kernel

$$
C_{\text {kernel }}\left(x_{1}, x_{2}\right)=\sigma_{k_{C}}^{2} \exp \left(\frac{-\left|x_{1}-x_{2}\right|}{b}\right)
$$

where the coefficient of variation $\sigma_{k_{C}}^{2}$ and correlation length $b$ are stated in Table 1.

Table 1. Parameters used in analysis

\begin{tabular}{|l|c|}
\hline \multicolumn{1}{|c|}{ Parameter } & Value \\
\hline \hline $\begin{array}{l}\text { Heat capacity per unit volume } \\
\text { (deterministic) }\end{array}$ & $c=434.0 \mathrm{~J} /(\mathrm{kgK})$ \\
\hline Density (deterministic) & $k_{F}=54 \mathrm{~W} /(\mathrm{mK})$ \\
\hline $\begin{array}{l}\text { Fourier thermal conductivity } \\
\text { (deterministic) }\end{array}$ & $\left\langle k_{C}(x, \omega)\right\rangle=1210.0 \cdot 10^{10} \mathrm{~W} /(\mathrm{mKs})$ \\
\hline $\begin{array}{l}\text { Cattaneo thermal conductivity } \\
\text { random - mean) }\end{array}$ & $\sigma_{k_{C}}^{2}=2500.0 \cdot 10^{10} \mathrm{~W} /(\mathrm{mKs})$ \\
\hline $\begin{array}{l}\text { Cattaneo thermal conductivity } \\
\text { (random - coefficient of variance) }\end{array}$ & $\tau=20.0 \cdot 10^{-12} \mathrm{~s}$ \\
\hline Relaxation time & $\alpha_{c}=9.0 \mathrm{~W} /\left(\mathrm{m}^{2} \mathrm{~K}\right)$ \\
\hline $\begin{array}{l}\text { Heat convection coefficient } \\
\text { (deterministic) }\end{array}$ & $\varepsilon_{r}=0.625$ \\
\hline Emissivity (deterministic) & $\sigma_{B}=5.67 \cdot 10^{-8} \mathrm{~W} /\left(\mathrm{m}^{2} \mathrm{~K}^{4}\right)$ \\
\hline Stefan-Boltzmann constant & $b=0.005 \mathrm{~m}^{4}$ \\
\hline Thickness & $b=0.001 \mathrm{~m}$ \\
\hline Correlation length & \\
\hline
\end{tabular}

Using the Karhunen-Loève expansion, the Cattaneo thermal conductivity can be written in the form

$$
k_{C}(x, \omega) \approx\left\langle k_{C}(x, \omega)\right\rangle+\sum_{i=1}^{M} \xi_{i}(\omega) \sqrt{\lambda_{i}} f_{i}(x)
$$


Therefore, the random matrix present in equation (3.14) can be expressed as

$$
\begin{aligned}
& \mathbf{F}_{x=0}^{\text {expand }}\left(x=0, \mathbf{T}_{\text {spect }}^{\text {vector }}(t)\right) \\
& \quad=\left\langle\boldsymbol{\Phi}(\boldsymbol{\xi})\left(\left(\mathbf{F}_{x=0}(x=0)\right)_{0} \xi_{0}+\sum_{i_{F}=1}^{M_{F}} \xi_{i}\left(\mathbf{F}^{0}(x=0)\right)_{i_{F}}\right) f_{F}\left(\left(\mathbf{T}_{\text {spect }}^{\text {vector }}(t)\right)^{\mathrm{T}} \boldsymbol{\Phi}(\boldsymbol{\xi})\right)\right\rangle
\end{aligned}
$$

This matrix takes non-zero values for the boundary node $(x=0)$. The matrices $(\mathbf{F}(x))_{0}=\mathbf{0}$, $\left(\mathbf{F}^{0}(x)\right)_{i_{F}}=\mathbf{0}$ are generally equal to zero, only for $x=0\left(\mathbf{F}_{x=0}(x=0)\right)_{0}=\mathbf{I}$. For the mixed boundary condition (convection-radiation) it can be assumed that (parameters in Table 1)

$$
\begin{aligned}
& f_{F}\left(\left(\mathbf{T}_{\text {spect }}^{\text {vector }}(t)\right)^{\mathrm{T}} \boldsymbol{\Phi}(\boldsymbol{\xi})\right)=\alpha_{c}\left(T_{\text {pulse }}(t)-\left(\left(\mathbf{T}_{\text {spect }}^{\text {vector }}(t)\right)^{\mathrm{T}} \mathbf{\Phi}(\boldsymbol{\xi})\right)\right) \\
& \quad+\varepsilon_{r} \sigma_{B}\left[T_{\text {pulse }}(t)^{4}-\left(\left(\mathbf{T}_{\text {spect }}^{\text {vect }}(t)\right)^{\mathrm{T}} \boldsymbol{\Phi}(\boldsymbol{\xi})\right)^{4}\right]
\end{aligned}
$$

Because the Cattaneo thermal conductivity is independent of the temperature function $f_{K}$ included in the matrix

$$
\begin{aligned}
& \mathbf{K}^{\text {expand }}\left(x, \mathbf{T}_{\text {spect }}^{\text {vector }}(t)\right) \\
& \quad=\left\langle\boldsymbol{\Phi}(\boldsymbol{\xi})\left((\mathbf{K}(x))_{0} \xi_{0}+\sum_{i_{K}=1}^{M_{K}} \xi_{i_{K}}\left(\mathbf{K}^{0}(x)\right)_{i_{K}}\right) f_{K}\left(\left(\mathbf{T}_{\text {spect }}^{\text {vect }}(t)\right)^{\mathrm{T}} \mathbf{\Phi}(\boldsymbol{\xi})\right)(\boldsymbol{\Phi}(\boldsymbol{\xi}))^{\mathrm{T}}\right\rangle
\end{aligned}
$$

(6.6) can be written as $f_{K}(\cdot)=1$, and the matrices $(\mathbf{K}(x))_{0},\left(\mathbf{K}^{0}(x)\right)_{i_{K}}$ can be determined from

$$
\begin{aligned}
& (\mathbf{K}(x))_{0}=\int_{\mathbb{D}} \nabla \mathbf{N} k_{F} \nabla \mathbf{N}^{\mathrm{T}} d x+\int_{\mathbb{D}} \nabla \mathbf{N}\left\langle k_{C}(x, \omega)\right\rangle \nabla \mathbf{N}^{\mathrm{T}} d x \\
& \left(\mathbf{K}^{0}(x)\right)_{i_{K}}=\int_{\mathbb{D}} \nabla \mathbf{N}\left(\sqrt{\lambda_{i_{K}}} f_{i_{K}}(x)\right) \nabla \mathbf{N}^{\mathrm{T}} d x
\end{aligned}
$$

the proposed modified SSFEM has been compared to the Monte Carlo method using the C- and F-process models. As the relevant set of points, the heated surface has been chosen. As shown in Fig. 2, there is a good correlation between the methods. SSFEM is giving smaller values for the

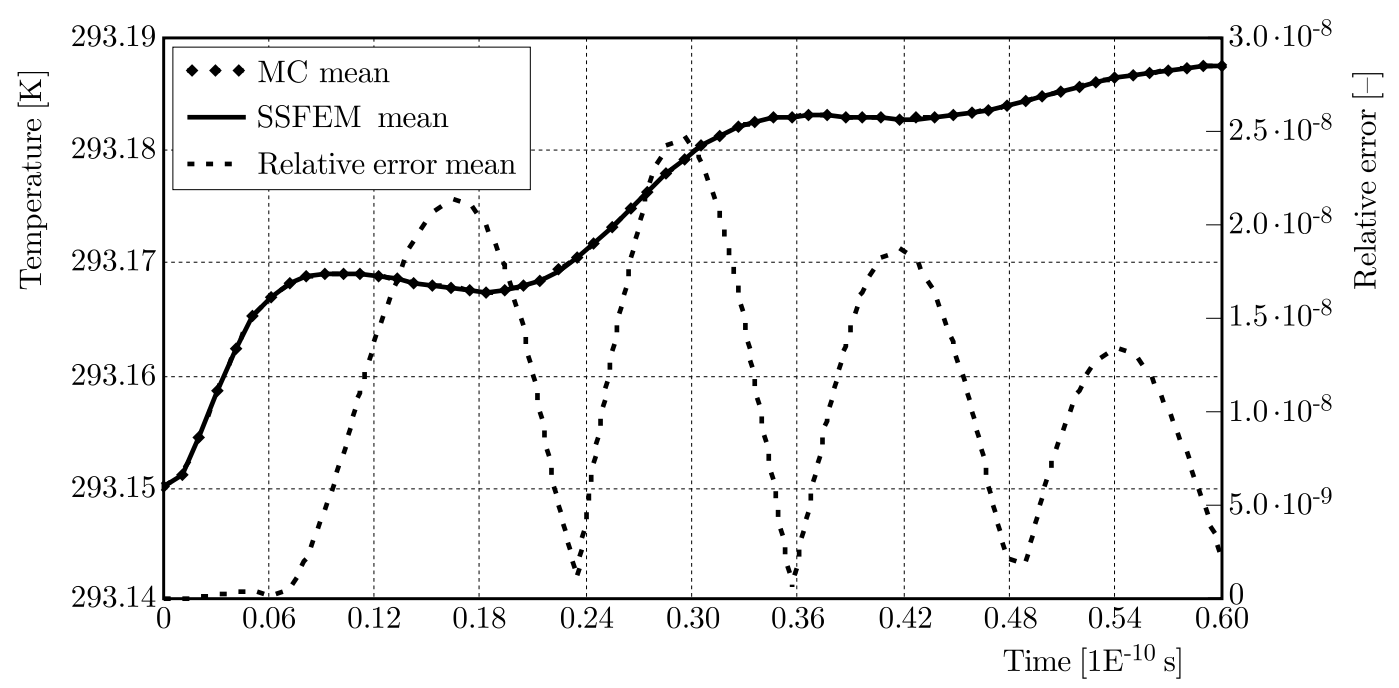

Fig. 2. Temperature mean value and relative error between SSFEM (3rd order polynomial chaos, 2nd order Karhunen-Loève expansion) and Monte Carlo (5000 samples) solution in function of time at the heated surface 
standard deviation than MC (Fig. 3), which is typical for this method (Ghanem and Spanos, 2003). It can be seen that the biggest relative error for the standard deviation occurs in time nodes with smallest values. The relative error of temperature (Fig. 2) between the mean value obtained from the SSFEM and Monte Carlo solution is small with the extremum not exceeding $2.5 \cdot 10^{-8}$. Computations have been performed for $P=3$ order of polynomial chaos and $M=2$ order of Karhunen-Loève expansion.

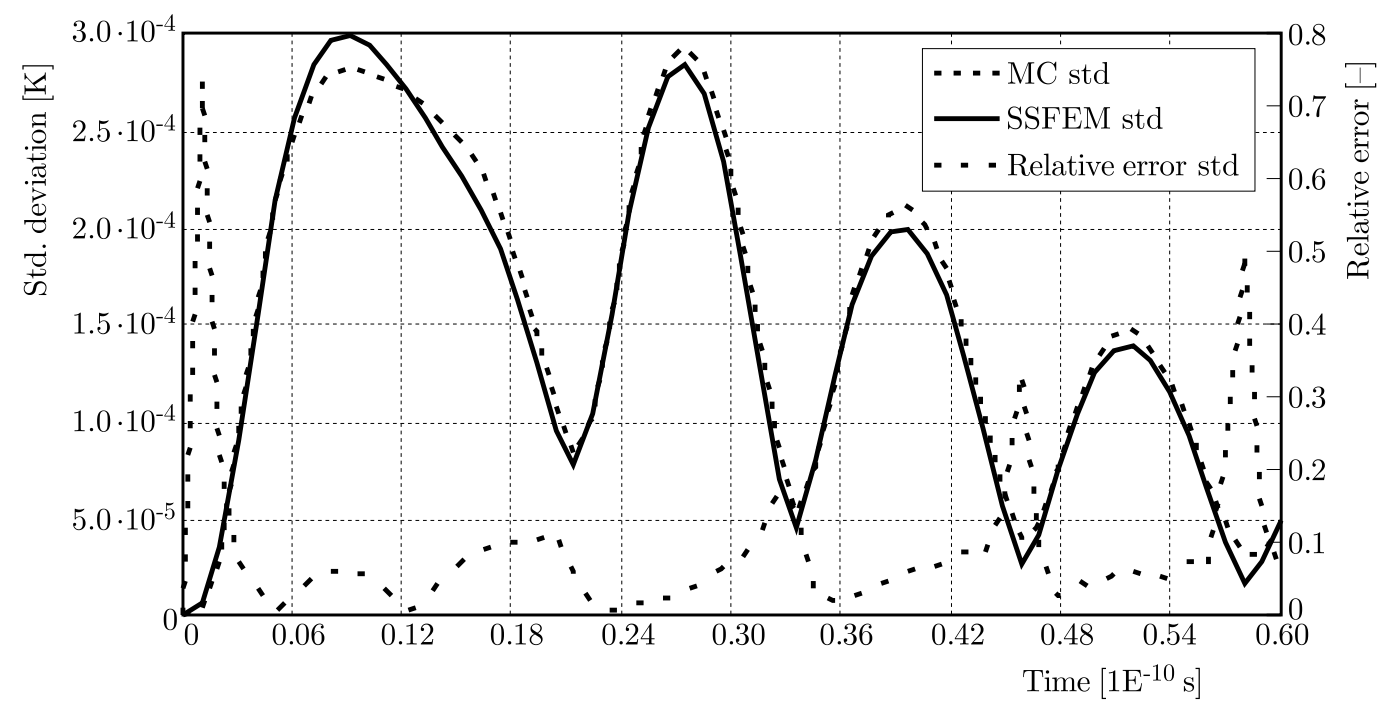

Fig. 3. Temperature standard deviation and relative error between SSFEM (3rd order polynomial chaos, 2nd order Karhunen-Loève expansion) and Monte Carlo (5000 samples) solution in function of time at the heated surface

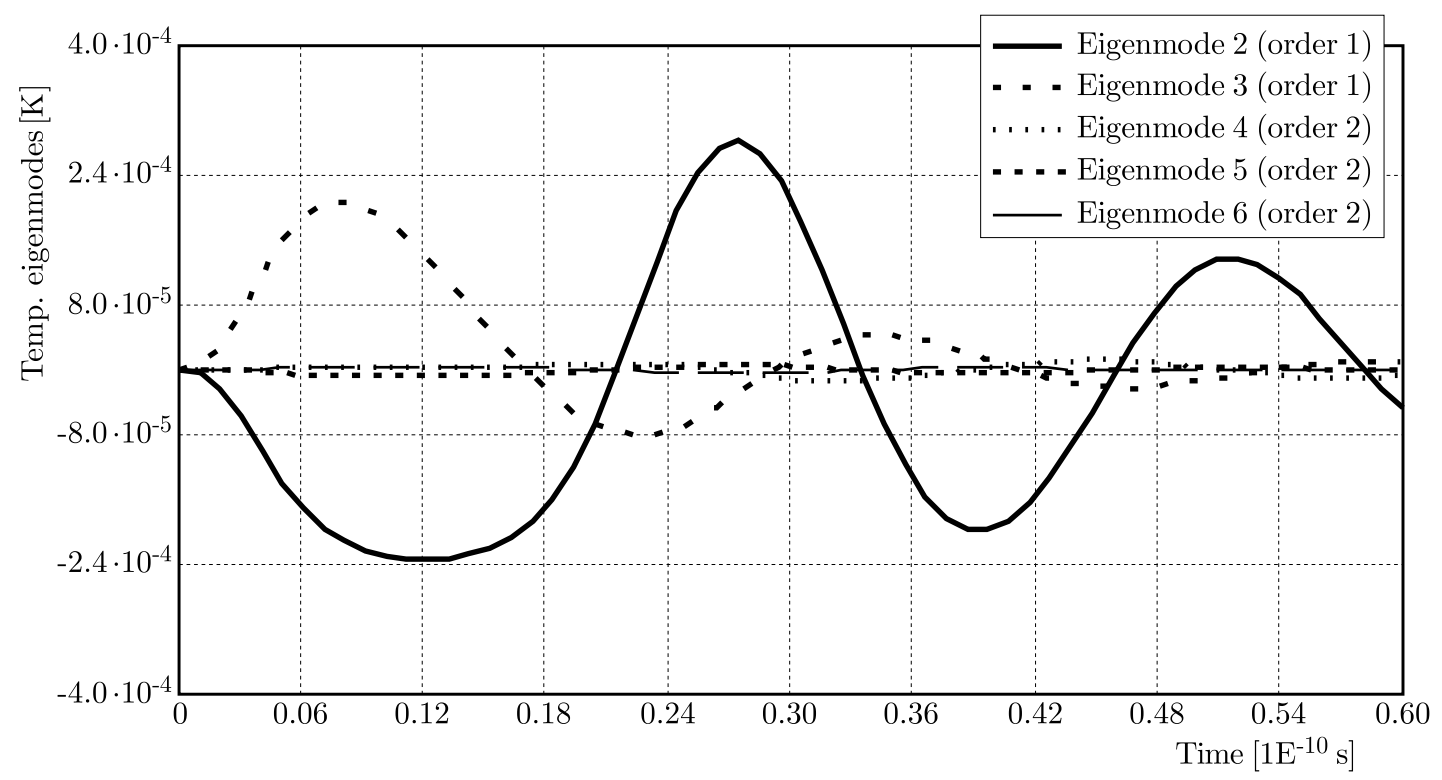

Fig. 4. Temperature eigenmodes obtained from SSFEM solution in function of time at the heated surface - 1st and 2nd order of polynomial chaos, 2nd order Karhunen-Loève expansion

Figure 4 illustrates solutions for successive orders of polynomial chaos. As can be seen, the biggest influence is the first order of the expansion on the basis of which it can be concluded that the improvement of the convergence of statistical moments by increasing the order of expansion in polynomial chaos will be small. 


\section{Concluding remarks}

The paper develops an approach to analysis stochastic nonlinear partial differential equations (SPDE's). As an example of stochastic analysis, the heat waves equation has been shown. The C-F-processes constitutive model has been chosen for the analysis. It can be reduced to the Fourier, Cattaneo and Jeffery types of models. A modified SSFEM, which consists in the separation of dependent and independent variables in the main matrices, has been proposed to solve nonlinear governing equations. The modified SSFEM has been compared to the Monte Carlo method. The comparison has shown that the proposed method works with nonlinear problems well and for equation (4.1) the solutions generated by the SSFEM method are convergent to solutions generated by the Monte Carlo method due to the first and second statistical moment. The analysis has revealed that the largest difference in the results obtained from the SSFEM and MC method is generated in time nodes with the smallest standard deviation (local minima). Comparison of the results from the methods has aimed at demonstrating compliance rather than efficiency or time consumption of the SSFEM. In order to check time consumption of the method in relation to MC or SCM, one should use the domain decomposition method (Subber and Sarkar, 2014) and methods of reducing the integration time of the main matrices (e.g. Smolyak sparse grid method (Smolyak, 1963)) which would allow one to use parallel processing.

\section{References}

1. Acharjee S., Zabaras N., 2006, Uncertainty propagation in finite deformations - a spectral stochastic Lagrangian approach, Computer Methods in Applied Mechanics and Engineering, 195, $19,2289-2312$

2. Acharjee S., Zabaras N., 2007, A non-intrusive stochastic Galerkin approach for modeling uncertainty propagation in deformation processes, Computational Stochastic Mechanics, 85, 5, $244-254$

3. Al-NimR, M., 1997, Heat transfer mechanisms during short-duration laser heating of thin metal films, International Journal of Thermophysics, 18, 5, 1257-1268

4. Arregui-Mena J.D., Margetts L., Mummery P.M., 2016, Practical application of the stochastic finite element method, Archives of Computational Methods in Engineering, 23, 1, 171-190

5. Babuška I., Nobile F., Tempone R., 2007, A stochastic collocation method for elliptic partial differential equations with random input data, SIAM Journal on Numerical Analysis, 45, 3, 1005-1034

6. Bargmann S., Favata A., 2014, Continuum mechanical modeling of laser-pulsed heating in polycrystals: a multi-physics problem of coupling diffusion, mechanics, and thermal waves, ZAMMJournal of Applied Mathematics and Mechanics/Zeitschrift für Angewandte Mathematik und Mechanik, 94, 6, 487-498

7. Bathe K.J., 1996, Finite Element Procedures, Prentice Hall, New Jersey

8. Cattaneo M., 1948, Sulla Conduzione de Calor, Mathematics and Physics Seminar, 3, 3, 83-101

9. Fourier J., 1822, Theorie Analytique de la Chaleur, Chez Firmin Didot, Paris

10. Ghanem R.G., Spanos P.D., 2003, Stochastic Finite Elements: A Spectral Approach, Dover Publications, Mineola, USA

11. Ghosh D., Avery P., Farhat C., 2008, A method to solve spectral stochastic finite element problems for large-scale systems, International Journal for Numerical Methods in Engineering, 00, $1-6$

12. Hu J., Jin S., XIU, D., 2015, A stochastic Galerkin method for Hamilton-Jacobi equations with uncertainty, SIAM Journal on Scientific Computing, 37, 5, A2246-A2269 
13. Joseph D.D., Preziosi L., 1989, Heat waves, Reviews of Modern Physics, 61, 1, 41-73

14. Kamiński M., 2013, The Stochastic Perturbation Method for Computational Mechanics, John Wiley \& Sons, Chichester

15. Le Maitre O.P., Knio O.M., 2010, Spectral Methods for Uncertainty Quantification: with Applications to Computational Fluid Dynamics, Springer Science \& Business Media, Doredrecht

16. Matthies H.G., Keese A., 2005, Galerkin methods for linear and nonlinear elliptic stochastic partial differential equations, Computer Methods in Applied Mechanics and Engineering, 194, 2, $1295-1331$

17. Nouy A., 2008, Generalized spectral decomposition method for solving stochastic finite element equations: invariant subspace problem and dedicated algorithms, Computer Methods in Applied Mechanics and Engineering, 197, 51, 4718-4736

18. Nouy A., Le Maitre O.P., 2009, Generalized spectral decomposition for stochastic nonlinear problems, Journal of Computational Physics, 228, 1, 202-235

19. SŁużAleC A., 2003, Thermal waves propagation in porous material undergoing thermal loading, International Journal of Heat and Mass Transfer, 46, 9, 1607-161

20. Smolyak S.A., 1963, Quadrature and interpolation formulas for tensor products of certain classes of functions, Doklady Akademii Nauk SSSR, 4, 240-243

21. Stefanou G., 2009, The stochastic finite element method: past, present and future, Computer Methods in Applied Mechanics and Engineering, 198, 9, 1031-1051

22. Stefanou G., Savvas D., Papadrakakis M., 2017, Stochastic finite element analysis of composite structures based on mesoscale random fields of material properties, Computer Methods in Applied Mechanics and Engineering, 326, 319-337

23. Straughan B., 2011, Heat Waves, Springer, New York

24. Subber W., Sarkar A., 2014, A domain decomposition method of stochastic PDEs: An iterative solution techniques using a two-level scalable preconditioner, Journal of Computational Physics, 257, 298-317

25. Tамма K.K., Zhou X., 1998, Macroscale and microscale thermal transport and thermo-mechanical interactions: some noteworthy perspectives, Journal of Thermal Stresses, 21, 3-4, 405-449

26. VÁN P., FülöP T., 2012, Universality in heat conduction theory: weakly nonlocal thermodynamics, Annalen der Physik, 524, 8, 470-478

27. Vernotte P., 1958, Les paradoxes de la theorie continue de l'equation de la chaleur, Comptes Rendus, 246, 3154-3155

28. XIU D., 2010, Numerical Methods for Stochastic Computations: A Spectral Method Approach, Princeton University Press, Princeton

29. Xiu D., Hesthaven J.S., 2005, High-order collocation methods for differential equations with random inputs, SIAM Journal on Scientific Computing, 27, 3, 1118-1139

30. XiU D., Karniadakis G.E., 2003, A new stochastic approach to transient heat conduction modeling with uncertainty, International Journal of Heat and Mass Transfer, 46, 24, 4681-4693

31. Zakian P., Khaji N., 2016, A novel stochastic-spectral finite element method for analysis of elastodynamic problems in the time domain, Meccanica, 51, 4, 893-920 